\title{
Autopsy Review of Sudden Cardiovascular Deaths In A Tertiary Hospital At South Eastern Nigeria From July 2007-June 2012.
}

\author{
Nnoli M.A.*,Nwafor C.C **,Jegede O.O**,Nwabuko C.O***,Abalichuku**** \\ *University of Calabar,Dept of Anatomic \& Forensic Pathology, Calabar. \\ **Federal Medical Centre,Dept of AnatomicPathology,Umuahia.Abia. \\ ***Federal Medical Centre,Dept of Haematology,Umuahia.Abia. \\ ****Federal Medical Centre,Dept Of Ophthalmology,Umuahia.Abia.
}

\begin{abstract}
Aim and Objectives: To determine the cause of sudden cardiovascular deaths and observe morphological changes in heart following death.

Materials and Methods: A cross section of seventy-seven(77) cases of deaths involving only cardiovascular deaths autopsies from july 2007 to june 2012 was done. A detailed autopsy including external and internal postmortem examination was done. The results was analysed using Microsoft excel.

Results: These shows $62 \%$ of sudden cardiovascular deaths as males,38\% in females and $11 \%$ are of ages less than thirty(30) years. In this later age range males are $10 \%$ and females $1 \%$.

Conclusion: These goes to say if one could have identified all risk factors and measures instituted most patients would have been saved from sudden death.

Keywords: Sudden Cardiovascular deaths, Hypertension,autopsy
\end{abstract}

\section{Introduction:}

Cardiovascular disease is the most common cause of deaths in USA;it is the leading cause of deaths in men between 20-65 years of age. Zipes and wellens estimate that upto $80 \%$ of individuals dying suddenly of cardiac disease die of coronary artery disease. ${ }^{1}$ There is a circadian variation in the incidence in the early morning. Willich et al reported the peak incidence as between $7 \mathrm{am}$ and 9am (after discounting individuals found dead during this time), which was $70 \%$ higher than the average rate during the rest of the day. ${ }^{2}$ The explanation was attributed to increased activity of the sympathetic nervous system,known to occur in morning, which may predispose to cardiac arrhythmias.

Sudden cardiovascular deaths is currently described as natural,unexpected death occurring within an hour of onset of final symptoms. ${ }^{3}$ If sudden cardiovascular deaths were to occur in the young, a systematic forensic autopsy including toxicological analyses must be done. The toxicology is to exclude toxic causes and to determine any drug-related cardiomyopathy as cocaine or amphetamine induced cardiomyopathy which could lead to sudden death. Also cardiac toxicity of anabolic steroid abuse must also be taken into account. ${ }^{4}$

It is also well known that coronary artery disease (CAD) including acute myocardial infarction,recent thrombosis and high grade coronary stenosis $(>75 \%)$ due to atheroma is still major cause of death in people of 35 years. ${ }^{5}$

Pulmonary diseases are usually caused by pulmonary embolism and asthmatic attack. When neurological signs are elucidated it represents cerebral haemorrhages and epilepsy. ${ }^{3}$ There is now frequency of right ventricularcardiomyopathy (RVC in $70 \%$ of sudden death between ages of $20-40$ years in USA). ${ }^{6}$

The new forms of RVC with minimal gross anomalies and other exclusive left ventricle involvement have been described. ${ }^{7,8}$ These has shown that left ventricle and interventricular septum involvement has a poor prognosis. Clinically,it has been shown also that patients with left ventricular hypertrophy $(\mathrm{LVH})$ have significantly more premature contractions than normal individuals or those with hypertension without (LVH) ${ }^{9-11}$ In most cases,sudden cardiovascular deaths have been recorded from coronary artherosclerosis. In study of 500 consecutive autopsies of ages 20-99 years Dimaio et al found acute thrombosis in $13.4 \%$ as cause of death. ${ }^{12}$ The left coronary and its branches showed higher incidence of thrombosis when compared with right. Hence it is acceptable to see in all deaths due to coronary artherosclerosis severe artherosclerosis of the coronary vessels. However, this is obscured in hypertensive cardiovascular disease as this is lacking because of plaque formation resulting to thickening of the walls by artheriosclerotic deposits. In elderly the lumina are patent,vessels rigid, calcified tubes because of calcium deposits in vessel walls. In some others while epicardial coronary arteries are non-occluded,microscopic examination of myocardium shows severe occlusive dysplasia of the intramural coronary arteries. ${ }^{13}$

Berry aneurysms per se are not uncommon as few cases seen were with hypertension and a social history of smoking which could have an effect in formation of aneurysm. The co-existing artherosclerosis may 
play a second role leading to focal destruction and weakening of the vessel walls. Multiplicity of aneurysm most invariably rupture at apex with haemorrhages into subarachnoid space. ${ }^{14.15}$

Heart failure has become an increasingly prevalent cause of death world-wide despite advances in therapy still cause sudden unexpected deaths. ${ }^{16}$

\section{Material And Methods:}

the study was carried out in a tertiary hospital at south east of Nigeria from July 2007 -June 2012. A total of 77 bodies of both sexes,aged and young were autopsied. The result was analyzed using all information obtained in medical case history. These includes all particulars of the deceased,activity prior to death,past medical/social history(smoking habit,known hypertension or diabetic);results of any investigation;any pathology in organs other than cardiovascular system. Any eye witness account was consulted prior to death.

III. Result:

CARDIOVASCULAR DEATH IN JULY 2007- JUNE 2012

\begin{tabular}{|l|l|l|l|l|l|}
\hline $\mathbf{2 0 0 7}$ & $\mathbf{2 0 0 8}$ & $\mathbf{2 0 0 9}$ & $\mathbf{2 0 1 0}$ & $\mathbf{2 0 1 1}$ & $\mathbf{2 0 1 2}$ \\
\hline 56 & 68 & 40 & 75 & 29 & 70 \\
\hline & 88 & 45 & 50 & 70 & 65 \\
\hline & 34 & 27 & 55 & 54 & 55 \\
\hline & 36 & 27 & 55 & 25 & 60 \\
\hline & 72 & 70 & 25 & 45 & 75 \\
\hline & 36 & 40 & 33 & & 49 \\
\hline & 72 & 45 & 72 & & 50 \\
\hline & & 27 & 25 & & 48 \\
\hline & & 25 & 20 & & 35 \\
\hline & & 89 & 50 & & 68 \\
\hline & & 14 & 60 & & 57 \\
\hline & & Adult & 25 & & 60 \\
\hline & & Adult & Adult & & 48 \\
\hline & & 54 & 53 & & 63 \\
\hline & & Adult & 50 & & 56 \\
\hline & & 18 & 22 & & 54 \\
\hline & & 70 & & & 78 \\
\hline & & 40 & & & 64 \\
\hline & & & & & 76 \\
\hline & & & & & 72 \\
\hline & & & & & 60 \\
\hline & & & & & 72 \\
\hline & & & & & 47 \\
\hline & & & & & 45 \\
\hline & & & & & 39 \\
\hline & & & & & 43 \\
\hline & & & & & 44 \\
\hline & & & & & \\
\hline & & & & & \\
\hline
\end{tabular}

GENERAL OVERVIEW OF AGE DISTRIBUTION OF CARDIOVASCULAR DEATH FROM JULY 2007- JUNE 2012

\begin{tabular}{|l|l|l|l|l|l|l|l|l|l|l|l|}
\hline $\mathbf{2 0 0 7}$ & \multicolumn{3}{l}{$\mathbf{2 0 0 8}$} & \multicolumn{2}{l|}{$\mathbf{2 0 0 9}$} & $\mathbf{2 0 1 0}$ & \multicolumn{2}{l|}{$\mathbf{2 0 1 1}$} & \multicolumn{2}{l|}{$\mathbf{2 0 1 2}$} \\
\hline Male & Female & Male & Female & Male & Female & Male & Female & Male & Female & Male & Female \\
\hline 0 & 56 & 68 & 0 & 27 & 40 & 75 & 20 & 29 & 25 & 70 & 65 \\
\hline & & 88 & & 45 & 40 & 50 & 55 & 70 & & 55 & 60 \\
\hline & & 34 & & 27 & 89 & 55 & & 54 & & 49 & 75 \\
\hline & & 68 & & 70 & 14 & 55 & & & & 35 & 50 \\
\hline & & 36 & & 45 & & 25 & & & & 68 & 48 \\
\hline
\end{tabular}


Autopsy Review Of Sudden Cardiovascular Deaths In A Tertiary Hospital At South Eastern Nigeria

\begin{tabular}{|l|l|l|l|l|l|l|l|l|l|l|l|}
\hline & & 72 & & 27 & & 50 & & & & 57 & 60 \\
\hline & & & & 25 & & 33 & & & & 78 & 48 \\
\hline & & & & 54 & & 72 & & & & 64 & 63 \\
\hline & & & & 18 & & 50 & & & & 72 & 56 \\
\hline & & & & & & 25 & & & & 45 & 56 \\
\hline & & & & & & 50 & & & & 39 & 54 \\
\hline & & & & & & 60 & & & & 40 & 76 \\
\hline & & & & & & 53 & & & & 43 & 60 \\
\hline & & & & & & 25 & & & & 44 & 72 \\
\hline & & & & & & 22 & & & & 76 & 47 \\
\hline & & & & & & & & & & & 80 \\
\hline
\end{tabular}
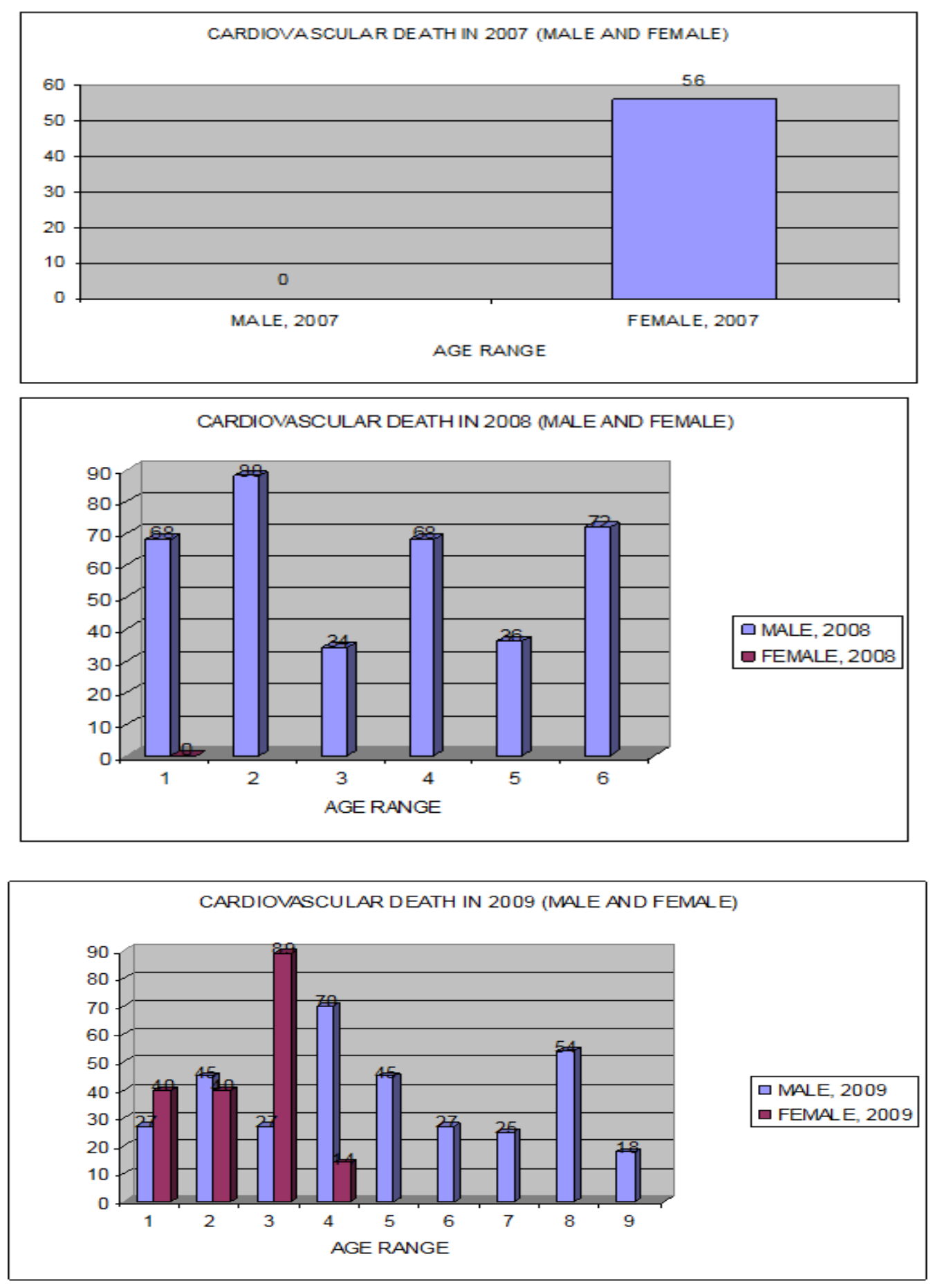

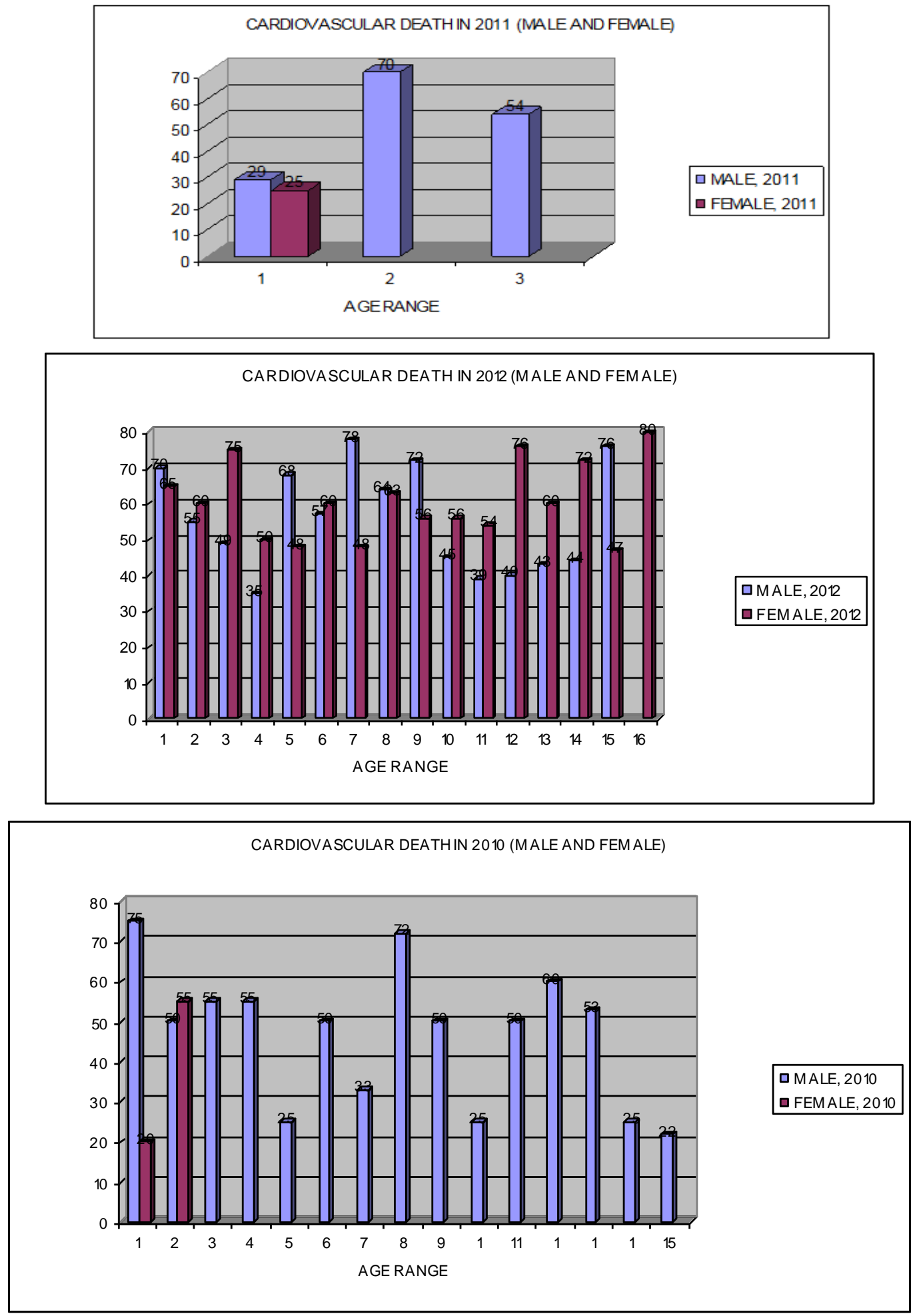

\section{Discussion:}

Sudden death is currently described as natural unexpected death occurring within an hour of new symptoms. Most studies focused on cardiac causes of death because they are always cardiovascular related. This is because cardiac causes are leading cause of sudden death,as other causes are not well known since many such deaths are not autopsied.

In most of the hospitalized patients with a known history who died suddenly,autopsy revealed an enlarged heart with left ventricular hypertrophy and minimal coronary artherosclerosis. ${ }^{17}$ The mechanism of death in these cases is sudden cardiac arrhythmia most likely ventricular fibrillation. These have been seen clinically that patients with left ventricular hypertrophy have more ventricular premature contractions than 
normal individuals or ones with hypertension without left ventricular hypertrophy. ${ }^{9-11}$ This observation agrees with our study that a significant number of individuals who die suddenly and unexpected with clinical history of hypertension have only left ventricular hypertrophy without severe artherosclerotic involvement of their coronary arteries.

A few of our patient had a ruptured berry aneurysms invariably at the apex. These led to haemorrhage into the subarachnoid spaces and in the substance of the brain. Death here is due to generalized vasospasm triggered by the subarachnoid haemorrhage, with resultant ischaemic injury to the brain.

In our study, $62 \%$ of deaths recorded were males, $38 \%$ females and $30 \%$ below the age of 30 years. In this age range still male were $10 \%$ and $1 \%$ are female. This shows the great preponderance of the cases that occurred were in males. Also most of the male middle aged as seen in the graphical representation died as a result of sudden death rupture of cerebral vessels leading to intracerebralhaemorrhage. This is supported by Vincent dimaio et $\mathrm{al}^{18}$ when he said that intracerebralhaemorrhages are more common in males and in negroid race than in whites probably due to greater incidence of hypertension.

The sudden death seen in the young is upmost important despite our detailed forensic autopsy though lacking forensic toxicological analysis. Indeed, toxicology is important as to exclude a toxic causesand help to determine drug related cardiomyopathy as cocaine or amphetamine-induced cardiomyopathy which can give sudden death. Hair testing is needed even if no or low levels of drug are detected in blood,inorder to show a past history of drug abuse. These result must be compared with a known cardiac pathologic findings suggestive of cocaine or amphetamine cardiac toxicity, as association of microfocal fibrosis,contraction band necrosis and cardiomyocyte hypertrophy. Also cardiac toxicity of anabolic steroids must be checked. ${ }^{4}$

STUDY LIMITATIONS: These include the relatively low patronage to autopsy and varying degrees of description/detail autopsy reports. This is attributed to the attitudinal/ignorance of the relatives and at times the unwillingness of the clinicians to request for autopsy. They general belief in both relatives/clinician in most cases is patient is a known hypertension hence it is not necessary. They few that accepted appear for knowledge purpose to guide their lineage/medico-legal attention in cases of feeling of medical negligence.

The sudden deaths seen in the young in our study no toxicologicanalysis were done. We known that a systemic forensic autopsy is never complete in this age group without toxicology analysis of drugs example cannabis, amphetamine etc.

\section{Conclusion:}

In conclusion, the progress in autopsy diagnosis of sudden death depends respectively on the following criteria- scene investigation, number and quality of autopsies and use of complementary technique especially molecular biology. Indeed, molecular autopsy is now needed to overcome autopsy diagnosis difficulties, although molecular investigation is not yet available in daily work of forensic pathologist. However, improvement needs to be done in all the new discovered methods of clinical,biological or imaging diagnosis used in investigating sudden deaths.

A major problem for cardiologist will be to identify assymptomatic patients at high risk of sudden death as to avoid early manifestation of the diseases. That is to develop preventive strategies as to use of anti-arryhthmic agents or implantable cardioverterdefribillator, and the ability to identify the incidence, causes and circumstances surrounding the sudden death must be well known and provided by the forensic pathologist. ${ }^{19}$

\section{References:}

[1] Zipes DP and Wellens HJJ. Sudden cardiac death. : Circulation 1988;8(21):2334-2351.

[2] WillichSN,LevyD,Rocco MB et al.: Circadian Variation in the incidence of sudden cardiac death in the Framingham heart study Population. Am Cardiol 1987;60:801-806.

[3] G.L. de la Grandmaison. Am. Durigon: Sudden adult death. a medico-legal series of 77cases between 1995 and 2000,med.sci.law 42 (2002) 225-232

[4] S.B.Karch :Pathology of Drug Abuse,rded.CRC Preas,2002,PP.183-187.

[5] P.Fornes. D. Lecomte, G.Nicolas : Sudden out-of-hospital coronay death in patients with no previous cardiac history: an analysis of 221 patients studied at autopsy,J.Foensic Sci.8 (1993) 1081-1084.

[6] F. Ahmad :The molecular genetics of arrhythmogenic right ventricular dysplasia-cardiomyopathy.Cli. invest.Med.26 (2003) 167178.

[7] M. Michalodimitrakis,A.Papadomanolakis,J.Stiakakis,K.Kanaki.:Left side /right ventricular cardiomyopathy, Med.Sci. Law 42 (2002) 313-317.

[8] A.P.Burke,S.Robinson,S.Radentz,J.Smialek,R.Virmani. Sudden death in right ventricular dysplasia with minimal gross abnormalities, J.Forensic Sci. 44(1990) 438-443

[9] MesserliFH,VenturaHO,Elizardi DJ et al: Hypertension and sudden death. AM J Med 1984;77:18-22.

[10] Haider Aw, Larson Mg,Benjamin EJ and Levy D : Increased Left ventricular mass and hypertrophy associated with increased risk for sudden death. JACC 1998;32(5):1454-9.

[11] FrolichED :Left ventricular hypertrophy and sudden death (Editorial Comment) JACC 1998;32(5):1460-2.

[12] Dimaio V.J.M and Dimaio DJM: Incidence of Coronary thrombosis in sudden death due to coronary artery disease. Am J Med \& Path 1993;14(4):273-5 
[13] Burke AP and Virmani R. : Intramural Coronary dysplasia of the ventricular septum and sudden death. Hum Path 1998;29(10):1124-7

[14] ChassonJL,Hundman WM : Berry aneurysms of the circle of willis. Neurology 1958;8:41-44.

[15] Freytag E: Fatal rupture of intracranial aneurysms : Arch Pathol 1966;81. 418-424.

[16] UretskyBF,Sheahan RG: Primary Prevention of sudden Cardiac death in heart failure: Will the solution be shocking? J AMCollCardiol. 1997; 30: 1589-1597.

[17] Kragol AH and Roberts WC: Sudden death and cardiomegaly unassociated with coronary,valvular congenital or specific myocardial disease. Am J Cardiol 1988; 61: 659-660.

[18] MesserliFH,VenturaHO,Elizardi DJ et al: Hypertension and sudden death. Am J. Med 1984;77: 18-22

[19] Vincent J Dimaio: Forensic Pathology $2^{\text {nd }}$ Edition. Deaths due to natural disease Pg 65.

[20] A. Martinez -Rubio,A. Bayes-Genis,J.Guindo,A. Bayes de Luna: Sudden cardiac death. Contrib. Sci. 1 (1999) 147-157. 